Fixed Point Theory, 20(2019), No. 1, 289-298

DOI: $10.24193 /$ fpt-ro.2019.1.19

http://www.math.ubbcluj.ro/ nodeacj/sfptcj.html

\title{
NONLINEAR CONTRACTION AND FUZZY COMPACT OPERATOR IN FUZZY BANACH ALGEBRAS
}

\author{
REZA SAADATI \\ Department of Mathematics, Iran University of Science and Technology \\ Tehran, Iran \\ E-mail: rsaadati@iust.ac.ir
}

\begin{abstract}
In this paper, at first, we consider the concept of fuzzy Banach algebras and fuzzy compact operators. Then we apply a fixed point theorem to solve the operator equation $A x B x=x$ in the fuzzy Banach algebras under a nonlinear contraction.

Key Words and Phrases: Banach algebra, fuzzy Banach space, nonlinear contraction, compact operator, completely continuous operator, Schauder fixed point theorem.

2010 Mathematics Subject Classification: $47 \mathrm{H} 10$.
\end{abstract}

Acknowledgement. The author is grateful to the reviewers for their valuable comments and suggestions.

\section{REFERENCES}

[1] R.P. Agarwal, Y.J. Cho, R. Saadati, On random topological structures, Abstr. Appl. Anal. 2011, Art. ID 762361, $41 \mathrm{pp}$

[2] C. Alsina, B. Schweizer, A. Sklar, Continuity properties of probabilistic norms, J. Math. Anal. Appl., 208(1997), 446-452.

[3] T. Bag, S.K. Samanta, Finite dimensional fuzzy normed linear spaces, J. Fuzzy Math., 11(2003), 687-705.

[4] T. Bag, S.K. Samanta, Fuzzy bounded linear operators, Fuzzy Sets and Systems, 151(2005), 513-547.

[5] A. Ben Amar, S. Chouayekh, A. Jeribi, New fixed point theorems in Banach algebras under weak topology features and applications to nonlinear integral equations, J. Funct. Anal., 259(2010), no. 9, 2215-2237.

[6] T. Bîzar, F. Pater, S. Nădăban, On fuzzy normed algebras, J. Nonlinear Sci. Appl., 9(9)(2016), $5488-5496$.

[7] S.C. Cheng, J.M. Mordeson, Fuzzy linear operators and fuzzy normed linear spaces, Bull. Calcutta Math. Soc., 86(1994), 429-436.

[8] B.C. Dhage, Remarks on two fixed point theorems involving the sum and the product of two operators, Comput. Math. Appl., 46(2003), 1779-1785.

[9] C. Felbin, Finite dimensional fuzzy normed linear spaces, Fuzzy Sets and Systems, 48(1992), 239-248.

[10] V. Gregori, S. Romaguera, Some properties of fuzzy metric spaces, Fuzzy Sets and Systems, 115(2000), 485-489.

[11] O. Hadžić, E. Pap, Fixed Point Theory in PM Spaces, Kluwer Academic Publishers, Dordrecht, 2001. 
[12] O. Hadžić, E. Pap, New classes of probabilistic contractions and applications to random operators, in: Y.J. Cho, J.K. Kim, S.M. Kong (Eds.), Fixed Point Theory and Application, vol. 4, Nova Science Publishers, Hauppauge, New York, 2003, pp. 97-119.

[13] B. Lafuerza-Guillén, A. Rodríguez-Lallena, C. Sempi, Normability of probabilistic normed spaces, Note Mat., 29(2009), no. 1, 99-111.

[14] A.K. Katsaras, Fuzzy topological vector spaces II, Fuzzy Sets and Systems, 12(1984), 143-154.

[15] I. Kramosil, J. Michalek, Fuzzy metric and statistical metric spaces, Kybernetica, 11(1975), 326-334.

[16] K. Mirmostafaee, Perturbation of generalized derivations in fuzzy Menger normed algebras, Fuzzy Sets and Systems, 195(2012), 109-117.

[17] S. Nădăban, Fuzzy continuous mappings in fuzzy normed linear spaces, Int. J. Comput. Commun. Control, 10(6)(2015), 834-842.

[18] S. Nădăban, I. Dzitac, Atomic decompositions of fuzzy normed linear spaces for wavelet applications, Informatica (Vilnius), 25(2014), no. 4, 643-662.

[19] D. O'Regan, R. Saadati, Nonlinear contraction theorems in probabilistic spaces, Appl. Math. Comput., 195(2008), 86-93.

[20] R. Saadati, J.H. Park, Intuitionistic fuzzy Euclidean normed spaces, Commun. Math. Anal., 1(2006), no. 2, 85-90.

[21] R. Saadati, S.M. Vaezpour, Some results on fuzzy Banach spaces, J. Appl. Math. Comput, $\mathbf{1 7}(2005), 475-484$.

[22] B. Schweizer, A. Sklar, Probabilistic Metric Spaces, Elsevier, North Holand, New York, 1983.

[23] G. Zhang, M. Zhang, On the normability of generalized Šerstnev PN spaces, J. Math. Anal. Appl., 340(2008), no. 2, 1000-1011.

Received: November 2, 2016; Accepted: May 18, $201 \%$. 
FUZZY BANACH ALGEBRAS 